\title{
LC-MS/MS Analysis of Sugars, Alditols, and Humectants in Smokeless Tobacco Products*
}

\author{
by \\ Liqun Wang, Stephen Stanfill, Liza Valentin-Blasini, Clifford H. Watson, and Roberto Bravo Cardenas \\ Department of Health and Human Services; U.S. Centers for Disease Control and Prevention; National Center for \\ Environmental Health; Division of Laboratory Sciences; Tobacco and Volatiles Branch, 4770 Buford Highway NE; \\ Atlanta 30341, GA, USA
}

\section{SUMMARY}

Globally, smokeless tobacco (ST) includes a wide array of chemically diverse products generally used in the oral cavity. Although ST has been widely investigated, this study was undertaken to determine the levels of sugars (mono- and di-saccharides), alditols, and humectants present in major ST categories/subcategories by using high performance liquid chromatography coupled with a triple quadrupole mass spectrometer (HPLC-MS/MS). The products studied included chewing tobacco (loose leaf, plug, twist), US moist snuff, Swedish snus, creamy snuff, dry snuff, dissolvable tobacco products, and tobacco-coated toothpicks. The highest mean sugar level was detected in chewing tobacco $(9.3-27.5 \%, w / w)$, followed by dissolvable tobacco $(2.1 \%)$; all other products were lower than $1 \%$. Creamy snuff had the highest mean alditol levels (22.6\%), followed by dissolvable tobacco (15.4\%); all others had levels lower than $1 \%$. The detected mean humectant levels ranged from non-detectable to $5.9 \%$. This study demonstrates the broad chemical diversity among ST. This research may aid researchers and public health advocates investigating the exposures and risks of ST. [Beitr. Tabakforsch. Int. 28 (2019) 203-213]

\section{KEYWORDS}

Smokeless tobacco (ST); chewing tobacco; snus; snuff; dissolvable tobacco; tobacco stick; creamy snuff; sugar; alditol; humectant; LC-MS/MS

\section{ZUSAMMENFASSUNG}

Allgemein umfasst der Begriff rauchloser Tabak (RT) eine große Vielfalt an chemisch unterschiedlichen Produkten, die in der Regel in der Mundhöhle konsumiert werden. Obwohl rauchloser Tabak bereits Gegenstand vieler Untersuchungen war, wurde diese Studie durchgeführt, um den Gehalt an Zuckern (Mono- und Disaccharide), Alditolen und Feuchthaltemitteln in wichtigen Kategorien bzw. Subkategorien rauchlosen Tabaks zu bestimmen. Dies geschah mithilfe der Hochleistungsflüssigkeitschromatographie gekoppelt mit einem Triple-Quadrupol-Massenspektrometer (HPLC-MS/MS). Die untersuchten Produkte waren Kautabak (Loose Leaf, Plug, Twist), Moist Snuff aus den USA, Swedischer Snus, Creamy Snuff, Dry Snuff, lösliche Tabakprodukte und Zahnstocher mit Tabaküberzug. Der höchste mittlere Zuckergehalt wurde in Kautabak gefunden $(9,3-27,5 \%, w / w)$, gefolgt von löslichem Tabak $(2,1 \%)$; bei allen übrigen Produkten lag der Gehalt bei unter $1 \%$. Creamy Snuff hatte den höchsten mittleren Alditolgehalt $(22,6 \%)$, gefolgt von löslichem Tabak (15,4\%); bei allen übrigen Produkten lag der Gehalt bei unter 1\%. Der mittlere festgestellte Gehalt an Feuchthaltemitteln reichte von nicht feststellbar bis zu 5,9\%. Diese Studie zeigt die große chemische Vielfalt bei den rauchlosen Tabakprodukten. Die Untersuchung kann Wissenschaftler und Mitarbeiter des öffentlichen Gesundheitswesens bei der Untersuchung von Exposition und Risiken durch rauchlosen Tabak unterstützen. [Beitr. Tabakforsch. Int. 28 (2019) 203-213] 


\section{RESUME}

Dans l'ensemble, le terme de tabac sans fumée (TSF) recouvre une large gamme de diverses substances chimiques consommées, en règle générale, par la cavité buccale. Malgré les vastes analyses déjà consacrées au TSF, la présente étude fut entamée dans le but de déterminer les niveaux de sucres (mono- et disaccharides), de sucres-alcools et d'humectants présents dans les principales catégories/sous-catégories de TSF à l'aide de la chromatographie en phase liquide à haute performance couplée à une spectrométrie de masse à triple quadripôle (HPLCMS/MS). Les produits analysés inclurent le tabac à chiquer (feuilles en vrac, tablette, corde), du tabac à priser humide américain, du snus suédois, du creamy snuff, du tabac à priser sec, des produits de tabac soluble et des cure-dents à la nicotine. Le niveau médian de sucres le plus élevé fut détecté dans le tabac à chiquer $(9,3-27,5 \%, \mathrm{p} / \mathrm{p})$, suivi du tabac soluble $(2,1 \%)$; tous les autres produits présentèrent des niveaux inférieurs à $1 \%$. Le creamy snuff présenta les niveaux médians de sucre-alcool les plus élevés $(22,6 \%)$, suivi du tabac soluble $(15,4 \%)$; tous les autres produits affichèrent des niveaux inférieurs à $1 \%$. Les niveaux médians d'humectants détectés oscillèrent entre nondétectable et 5,9\%. La présente étude démontre la grande diversité chimique parmi les formes de TSF et peut aider les chercheurs et les intervenants en faveur de la santé publique à explorer les degrés d'exposition et les risques du TSF. [Beitr. Tabakforsch. Int. 28 (2019) 203-213]

\section{INTRODUCTION}

Smokeless tobacco (ST) products are used broadly and their consumption is increasing in the US and some North European countries (1-4). These products are often promoted as a substitute for smoking tobacco products, mainly because of increased social acceptability, ease of use, including smoking-free environments, and perception of these products as reduced-harm products (5). A wide range of ST products, with different physical properties and uses, has increased in markets around the world. The major categories of ST include chewing tobacco, snus, snuff, dissolvable tobacco, creamy snuff, and tobacco sticks etc. $(1,4,6,7)$. Chewing tobacco, which is made from cured and often fermented tobacco, exists in several forms, such as loose leaf, plug, and twist. Snus, which is steam-cured, non-fermented and pasteurized tobacco, originated in Sweden and was first marketed in the US in 2006 (4). Snuff is produced consisting of air- and fire-cured tobacco and has two subcategories: moist and dry; these products are generally fermented. Dry snuff can be consumed by sniffing nasally or by placing in the mouth. Some snus and snuff products are packaged in a metal, plastic or cardboard 'tin' as loose tobacco; whereas, other products contain tobacco enclosed in a tea-bag like pouch for convenience and more discrete use. Dissolvable tobacco, introduced in the late 2000 s, contains non-fermented and finely processed tobacco that is pressed into strips, pellets (orbs), sticks that fully dissolve when being consumed. A similar product called tobacco stick consists of finely milled tobacco that is coated on toothpicks roughly $60 \mathrm{~mm}$ long. During use, the tobacco portion dissolves leaving the toothpick, which is discarded. Lastly, creamy snuff, a product from India, is a tobacco-containing paste sold in a toothpaste tube, mainly used by women in India as a dentifrice, presumably for oral health.

Researchers have found that ST use is associated with health problems, including heart and cardiovascular diseases (8-10), type 2 diabetes $(11,12)$, dental problems (13-15), and various cancers $(1,16-18)$. It is also known that poor oral health can contribute to heart disease (19-22). However, there are controversial findings on the ST's health effects $(5,16,23-25)$. The full impact of ST on public health is still unclear. Concerns have been raised as to whether ST facilitates smoking cessation or rather acts as a gateway to smoking or dual use $(24,26-28)$. For example, one study found that snus use was independently associated with increased risk of pancreatic cancer, but was unrelated to incidence of oral and lung cancer (16). Currently, more investigations and scientific proof are needed to get a better understanding of ST products and their potential health effects on users, and how to reduce the harmfulness of ST (5).

Various types of ST products contain different chemicals due to factors, such as tobacco origin, processing steps, such as curing, fermentation, aging, additives formulation, and storage conditions (29). It is known that air-, fire- and sun-cured tobacco typically has lower amounts of sugars than flue-cured tobacco (30). During fermentation, sugar content in tobacco can be impacted by the microbial metabolism as the levels of reducing sugars in tobacco can be depleted rapidly (31). Sugars exist in tobacco naturally and as an additive. Sugars and substances that contain sugars, such as molasses, honey, maple syrup, and fruit juices (apple, beet, fig, plum, etc.), are added to many tobacco products during tobacco processing. Therefore, different products may have varying health effects. Researchers have studied the health risk caused by sugars in ST $(14,15)$. TOMAR et al. concluded that chewing tobacco users had a higher risk of dental caries than did snuff users, smokers or non-smokers (15). The presence of sugar could affect the growth of some microbes and certain compounds these microbes may generate during use $(31,32)$. High sugar content of chewing tobacco may retard bacterial growth in storage; these bacteria are required for the metabolism of nitrosated, acylated, and oxidized pyridine alkaloids (32).

In previous studies in other laboratories, fructose, glucose, sucrose, maltose, and isomaltose were measured in 6 brands of chewing tobacco and 3 brands of moist snuff using a GC method (33). Using LC-MS/MS and IC methods, fructose, glucose, and sucrose were measured in 9 brands of chewing tobacco and 11 brands of snuff (34). The levels of humectants (glycerol, propylene glycol, and triethylene glycol) in 17 brands of moist snuff were measured using GC and GC-MS methods (35).

This work presents the sugar, alditol, and humectant profiles in 6 categories of ST in the current market, including chewing tobacco, snus, snuff, dissolvable tobacco, creamy snuff, and tobacco stick. This complements our previous work on the chemical characterization of ST about nicotine, $\mathrm{pH}$, and tobacco-specific $N$-nitrosamines (TSNAs) $(7,36,37)$. In this study, 5 sugars (fructose, glucose, 
mannose, sucrose, maltose), 3 alditols (xylitol, sorbitol, myo-inositol), and 3 humectants (glycerol, propylene glycol, and triethylene glycol) were measured using an LCMS/MS method previously developed and validated (38).

\section{MATERIALS AND METHODS}

\section{Chemicals}

Native standards for each of our analytes, D-(-)-fructose, D-(+)-glucose, D-(+)-mannose, D-(+)-maltose, sucrose, xylitol, D-sorbitol, glycerol, propylene glycol, and triethylene glycol, were purchased from Sigma-Aldrich (St. Louis, MO). Isotopically-labeled compounds used as internal standards, including D-fructose- ${ }^{13} \mathrm{C}_{6}$, D-glucose $-{ }^{13} \mathrm{C}_{6}, \mathrm{D}$ sucrose-glucose- $-{ }^{13} \mathrm{C}_{6}$, glycerol- ${ }^{13} \mathrm{C}_{3}$, and 1, 2-propanediol${ }^{13} \mathrm{C}_{3}$, were obtained from Cambridge Isotope Inc. (Andover, MA). The D-sorbitol- ${ }^{13} \mathrm{C}_{6}$ and LC-MS grade acetonitrile used in our study was obtained from Sigma-Aldrich (St. Louis, MO). J.T. Baker HPLC grade water and Fisher HPLC grade methanol were purchased from Fisher Scientific (Fairlawn, NJ, USA).

Individual standard stock solution and isotopically labeled internal standard stock solution were prepared in methanol/water $(1: 1, v / v)$, and stored at $-20{ }^{\circ} \mathrm{C}$. Working standard mixture solutions and internal standard mixture solutions were prepared in water and stored at $-20^{\circ} \mathrm{C}$. Ten standard solutions for the calibration curve were prepared in water before each analytical run.

\section{ST samples and sample preparation}

The ST examined in this study included the most popular US-made ST products: chewing loose (7 brands), chewing plug ( 5 brands), chewing twist ( 3 brands), moist snuff loose (6 brands), moist snus pouch (6 brands), dry snuff ( 7 brands), dissolvable strip (1 brand), dissolvable pellet (4 brands), dissolvable stick (1 brand), and tobacco stick (4 brands). In addition, we analyzed imported ST products: Creamy snuff from India and snus from Sweden, including moist snus pouch (6 brands) and moist snus loose (4 brands), and also 3 references of ST: CORESTA reference product moist snuff (CRP2), CORESTA reference product dry snuff (CRP3), and CORESTA reference product chewing loose (CRP4) (provided by the Tobacco Analysis Laboratory, North Carolina State University, Raleigh, NC, USA). All ST products were stored at $-70^{\circ} \mathrm{C}$ until further processing. For sample preparation, ST samples were removed from the freezer and placed in a refrigerator for a minimum of $24 \mathrm{~h}$. This will allow water to become thoroughly re-equilibrated throughout the ST samples. After removal from the refrigerator, the ST samples were allowed to equilibrate to ambient conditions before opening. Prior to sample extraction, loose leaf, plug, and twist ST were ground for 5 min using an electric spice grinder. Dissolvable pellets and sticks were cut into small pieces to obtain the required weight. From the tobacco sticks, the tobacco coating was scraped off using a scalpel. Sample weights of approximately $0.6 \mathrm{~g}$ (dry snuff, chewing tobacco, tobacco stick), $1.2 \mathrm{~g}$ (moist snuff, snus), or $0.1 \mathrm{~g}$ (dissolvable products) were weighed out in triplicates. A
17.5-mL aliquot of HPLC water was added to each sample. Samples were agitated at $250 \mathrm{rpm}$ on a Thermo Scientific MAXQ 2000 digital shaker (Fisher Scientific, Fairlawn, NJ, USA) at room temperature (approximately $22^{\circ} \mathrm{C}$ ) for $1 \mathrm{~h}$. A $500-\mu \mathrm{L}$ aliquot of the extract was transferred into vials and centrifuged at 5,000 rpm for $5 \mathrm{~min}$ (Legend Micro 17 Sorvall, Fisher Scientific, Fairlawn, NJ, USA). A 50.0- $\mu \mathrm{L}$ aliquot of supernatant and $50.0-\mu \mathrm{L}$ aliquot of working internal standard solution were mixed in a QSert Vial (SUPELCO, Sigma-Aldrich, St. Louis, MO, USA), then analyzed by LC-MS/MS. Two Quality Control-Low (QCL) and two Quality Control-High (QCH) samples were prepared and included in each analytical batch. Preparation of QCL and QCH was previously described by WANG et. al (38).

\section{LC-MS/MS Method}

An established LC-ESI-MS/MS method was applied for measuring the tobacco samples (38). In brief, an Agilent LC (Agilent Technology Inc., Wilmington, DE, USA), fitted with a Acquity UPLC BEH Amide column $(1.7 \mu \mathrm{m}$ particle size, $2.1 \mathrm{~mm}$ I.D. $\times 100 \mathrm{~mm}$, Waters Inc., Milford, MA, USA) was coupled with an API 5500 triple quad mass spectrometer (Applied Biosystems, Foster City, CA, USA). Samples were ionized using negative electrospray ionization (ESI) and introduced into the triple quad mass spectrometer operated under multiple-reaction monitoring (MRM) mode. Detailed LC conditions and mass parameters for all the analytes have been published elsewhere (38). Fructose and xylitol quantitation used D-fructose- ${ }^{13} \mathrm{C}_{6}$ as internal standard; glucose and mannose used D-glucose${ }^{13} \mathrm{C}_{6}$; sucrose, maltose, and myo-inositol used D-sucroseglucose- ${ }^{13} \mathrm{C}_{6}$; glycerol used glycerol- ${ }^{13} \mathrm{C}_{3}$; propylene glycol and triethylene glycol used 1, 2-propanediol- ${ }^{13} \mathrm{C}_{3}$; sorbitol used D-sorbitol- ${ }^{13} \mathrm{C}_{6}$. Data acquisition and analysis was performed using Analyst software (version 1.6.1, Applied Biosystems, Foster City, CA, USA). Relative response factor was calculated based on the ratio of the peak area of the analyte transition to that of the isotopically-labeled internal standard transition. The peak area ratio of the analyte transition to the isotopically-labeled internal standard transition was used to quantify the ST products by the calibration curve comparison.

\section{RESULTS AND DISCUSSIONS}

In the present study, 13 major categories/subcategories of domestic and imported ST were analyzed for 11 compounds, including sugars (mono- and disaccharides), alditols and humectants. One representative chromatograph from a US chewing plug is displayed in Figure 1. Levels of all analytes were expressed as the percentage of wet tobacco weight. Table 1 shows measurement results for each individual brand. Figure 2 compares mean total sugar, mean total alditol, and mean total humectants for the examined categories/subcategories. Table 2 presents the means and distribution ranges of total sugar, total alditol, and total humectant for each category or subcategory. Triethylene glycol was not detected in any of the analyzed products. 
Table 1. Levels of measured sugars, alditols, and humectants in selected smokeless tobacco products. Unit: \% (mg/mg)

\begin{tabular}{|c|c|c|c|c|c|c|c|c|c|c|c|c|c|c|}
\hline \multirow{2}{*}{$\begin{array}{c}\text { Category/ } \\
\text { Subcategory }\end{array}$} & \multirow{2}{*}{ Brand } & \multicolumn{5}{|c|}{ Sugars $^{a}$} & \multicolumn{3}{|c|}{ Alditols } & \multicolumn{2}{|c|}{ Humectants } & \multirow{2}{*}{$\begin{array}{c}\text { Total } \\
\text { Sugars }\end{array}$} & \multirow{2}{*}{$\begin{array}{c}\text { Total } \\
\text { Alditol }\end{array}$} & \multirow{2}{*}{$\begin{array}{c}\text { Total } \\
\text { Humectant }\end{array}$} \\
\hline & & Man & Fru & Glu & Suc & Mal & Xyl & Sor & Ino & Gly & PG & & & \\
\hline \multirow[t]{7}{*}{ US loose leaf } & 1 & 0.11 & 1.5 & 2.3 & 15.5 & 1.60 & 0.07 & 0.03 & 0.03 & 2.7 & 0.52 & 21.0 & 0.14 & 3.2 \\
\hline & 2 & 0.07 & 2.3 & 4.4 & 19.2 & 0.13 & 0.04 & 0.003 & 0.05 & 4.8 & $<$ LOD & 26.0 & 0.09 & 4.8 \\
\hline & 3 & 0.12 & 19.3 & 1.2 & 12.6 & 0.18 & 0.03 & 0.04 & 0.03 & 3.5 & $<$ LOD & 33.4 & 0.11 & 3.5 \\
\hline & 4 & 0.15 & 12.6 & 11.6 & 6.4 & 0.18 & 0.04 & 0.06 & 0.02 & 4.1 & 1.1 & 30.9 & 0.13 & 5.2 \\
\hline & 5 & 0.08 & 5.4 & 5.9 & 16.9 & 1.60 & 0.04 & 0.03 & 0.04 & 5.5 & 0.93 & 29.8 & 0.11 & 6.4 \\
\hline & 6 & 0.09 & 3.4 & 5.7 & 15.9 & 2.40 & 0.03 & 0.02 & 0.04 & 3.4 & 0.64 & 27.5 & 0.10 & 4.0 \\
\hline & 7 & 0.06 & 1.2 & 4.0 & 16.7 & 1.80 & 0.06 & 0.002 & 0.04 & 2.3 & 0.92 & 23.7 & 0.10 & 3.2 \\
\hline \multirow[t]{5}{*}{ US plug } & 1 & 0.15 & 2.7 & 3.4 & 13.3 & 2.50 & 0.05 & 0.004 & 0.05 & 3.3 & 0.65 & 22.0 & 0.10 & 3.9 \\
\hline & 2 & 0.08 & 1.3 & 3.2 & 16.0 & 0.12 & 0.07 & 0.01 & 0.04 & 3.0 & 0.96 & 20.7 & 0.11 & 4.0 \\
\hline & 3 & 0.09 & 2.9 & 2.6 & 13.7 & 0.10 & 0.06 & 0.92 & 0.09 & 5.8 & $<$ LOD & 19.3 & 1.10 & 5.8 \\
\hline & 4 & 0.08 & 3.0 & 2.8 & 13.3 & 0.11 & 0.06 & 0.15 & 0.09 & 5.8 & 1.3 & 19.3 & 0.30 & 7.1 \\
\hline & 5 & 0.31 & 6.9 & 5.1 & 5.3 & 1.90 & 0.06 & 0.05 & 0.11 & 2.1 & 1.1 & 19.5 & 0.22 & 3.2 \\
\hline \multirow[t]{3}{*}{ US twist } & 1 & 0.09 & 1.8 & 3.8 & 15.0 & $<$ LOD & 0.06 & 0.04 & 0.04 & 3.2 & 0.57 & 20.6 & 0.14 & 3.7 \\
\hline & 2 & $<$ LOD & $<$ LOD & $<$ LOD & 0.02 & 0.04 & 0.06 & 0.002 & 0.04 & 0.08 & $<$ LOD & 0.06 & 0.11 & 0.08 \\
\hline & 3 & 0.07 & 1.5 & 0.55 & 5.1 & $<$ LOD & 0.07 & 0.01 & 0.10 & 4.0 & $<$ LOD & 7.2 & 0.19 & 4.0 \\
\hline \multirow{6}{*}{$\begin{array}{l}\text { US moist } \\
\text { snuff (loose) }\end{array}$} & 1 & $<$ LOD & $<$ LOD & $<$ LOD & $<$ LOD & $<$ LOD & 0.03 & $<$ LOD & 0.03 & $<$ LOD & $<$ LOD & $<$ LOD & 0.06 & $<$ LOD \\
\hline & 2 & $<$ LOD & $<$ LOD & $<$ LOD & $<$ LOD & $<$ LOD & 0.002 & $<$ LOD & 0.05 & $<$ LOD & $<$ LOD & $<$ LOD & 0.05 & $<$ LOD \\
\hline & 3 & $<$ LOD & $<$ LOD & $<$ LOD & $<$ LOD & $<$ LOD & 0.005 & $<$ LOD & 0.05 & $<$ LOD & $<$ LOD & $<$ LOD & 0.06 & $<$ LOD \\
\hline & 4 & $<$ LOD & $<$ LOD & $<$ LOD & $<$ LOD & $<$ LOD & 0.04 & $<$ LOD & 0.02 & $<$ LOD & $<$ LOD & $<$ LOD & 0.06 & $<$ LOD \\
\hline & 5 & $<$ LOD & $<$ LOD & $<$ LOD & $<$ LOD & $<$ LOD & $<$ LOD & $<$ LOD & $<$ LOD & $<$ LOD & $<$ LOD & $<$ LOD & $<$ LOD & $<$ LOD \\
\hline & 6 & $<$ LOD & $<$ LOD & 0.05 & $<$ LOD & 0.004 & 0.01 & 0.53 & 0.05 & $<$ LOD & $<$ LOD & 0.05 & 0.59 & $<$ LOD \\
\hline \multirow{6}{*}{$\begin{array}{l}\text { US moist } \\
\text { snus (pouch) }\end{array}$} & 1 & $<$ LOD & $<\mathrm{LOD}$ & $<$ LOD & $<$ LOD & $<$ LOD & 0.01 & $<$ LOD & 0.06 & $<$ LOD & $<$ LOD & $<$ LOD & 0.07 & $<$ LOD \\
\hline & 2 & 0.01 & 0.01 & 0.10 & $<$ LOD & 0.01 & 0.01 & $<$ LOD & 0.05 & 0.004 & 0.69 & 0.14 & 0.06 & 0.69 \\
\hline & 3 & 0.01 & 0.003 & 0.04 & 0.08 & $<$ LOD & 0.13 & 0.01 & 0.16 & 0.09 & 2.9 & 0.14 & 0.30 & 3.0 \\
\hline & 4 & 0.01 & 0.01 & 0.05 & 0.06 & $<$ LOD & 0.09 & 0.004 & 0.14 & 0.07 & 2.5 & 0.12 & 0.23 & 2.6 \\
\hline & 5 & 0.01 & 0.01 & 0.04 & 0.05 & $<$ LOD & 0.10 & 0.004 & 0.14 & 0.08 & 2.7 & 0.11 & 0.24 & 2.8 \\
\hline & 6 & 0.02 & 0.38 & 0.24 & 1.00 & $<$ LOD & 0.04 & 0.002 & 0.08 & 0.05 & 6.0 & 1.7 & 0.12 & 6.0 \\
\hline \multirow{6}{*}{$\begin{array}{l}\text { SWE moist } \\
\text { snus (pouch) }\end{array}$} & 1 & 0.01 & 0.18 & 0.18 & 0.05 & 0.002 & 0.08 & 0.002 & 0.12 & 0.06 & 3.5 & 0.42 & 0.21 & 3.5 \\
\hline & 2 & 0.03 & 0.10 & 0.14 & 0.02 & 0.004 & 0.03 & 0.002 & 0.32 & 0.05 & 4.0 & 0.29 & 0.36 & 4.0 \\
\hline & 3 & 0.02 & 0.18 & 0.18 & 0.03 & 0.003 & 0.05 & 0.003 & 0.11 & 0.03 & 3.9 & 0.41 & 0.16 & 3.9 \\
\hline & 4 & 0.02 & 0.11 & 0.11 & 0.02 & $<$ LOD & 0.03 & 0.002 & 0.08 & 0.02 & 2.4 & 0.25 & 0.11 & 2.4 \\
\hline & 5 & 0.04 & 0.41 & 0.47 & 0.20 & 0.11 & 0.04 & 0.005 & 0.17 & 0.36 & 3.0 & 1.20 & 0.22 & 3.4 \\
\hline & 6 & 0.04 & 0.24 & 0.19 & 0.04 & 0.003 & 0.04 & 0.004 & 0.19 & 0.05 & 3.9 & 0.51 & 0.24 & 4.0 \\
\hline \multirow{4}{*}{$\begin{array}{l}\text { SWE moist } \\
\text { snus loose }\end{array}$} & 1 & 0.05 & 0.26 & 0.29 & 0.07 & 0.01 & 0.03 & 0.003 & 0.16 & 0.03 & 3.5 & 0.68 & 0.19 & 3.6 \\
\hline & 2 & 0.04 & 0.41 & 0.31 & 0.02 & $<$ LOD & 0.02 & 0.002 & 0.16 & 3.4 & 3.4 & 0.78 & 0.18 & 6.9 \\
\hline & 3 & 0.05 & 0.02 & 0.03 & 0.22 & 0.01 & 0.02 & 0.003 & 0.31 & 5.2 & 0.77 & 0.33 & 0.34 & 6.0 \\
\hline & 4 & 0.05 & 0.40 & 0.29 & 0.02 & 0.002 & 0.04 & 0.004 & 0.17 & 3.1 & 3.9 & 0.76 & 0.22 & 7.0 \\
\hline \multirow[t]{7}{*}{ US dry snuff } & 1 & 0.01 & 0.12 & 0.14 & 0.36 & 0.03 & 0.26 & $<$ LOD & 0.06 & 0.15 & $<$ LOD & 0.66 & 0.32 & 0.15 \\
\hline & 2 & 0.02 & 0.26 & 0.19 & 0.08 & 0.03 & 0.32 & $<$ LOD & 0.08 & 0.20 & $<$ LOD & 0.57 & 0.40 & 0.20 \\
\hline & 3 & 0.02 & 0.38 & 0.24 & 0.12 & 0.03 & 0.19 & $<$ LOD & 0.18 & 0.21 & $<$ LOD & 0.79 & 0.37 & 0.21 \\
\hline & 4 & 0.05 & 0.91 & 0.46 & 0.69 & 0.07 & 0.24 & 0.02 & 0.39 & 0.19 & $<$ LOD & 2.2 & 0.66 & 0.19 \\
\hline & 5 & 0.01 & 0.04 & 0.04 & 0.34 & 0.07 & 0.26 & $<$ LOD & 0.08 & 0.07 & $<$ LOD & 0.51 & 0.34 & 0.07 \\
\hline & 6 & 0.02 & 0.37 & 0.21 & 0.06 & $<$ LOD & 0.11 & $<$ LOD & 0.20 & 0.15 & $<$ LOD & 0.66 & 0.32 & 0.15 \\
\hline & 7 & 0.01 & 0.22 & 0.20 & 0.19 & 0.04 & 0.25 & $<$ LOD & 0.07 & 0.18 & $<$ LOD & 0.66 & 0.32 & 0.18 \\
\hline US dissolv- & pellet 1 & 0.04 & 0.33 & 0.27 & 0.13 & 0.36 & 0.02 & $<$ LOD & 0.20 & 0.05 & $<$ LOD & 1.1 & 0.22 & 0.05 \\
\hline & pellet 2 & 0.04 & 0.34 & 0.26 & 0.16 & 0.32 & 0.02 & $<$ LOD & 0.22 & 0.05 & $<$ LOD & 1.1 & 0.24 & 0.05 \\
\hline & pellet 3 & 0.11 & 1.40 & 1.20 & 0.90 & 0.09 & 0.02 & $<$ LOD & 0.21 & 0.05 & $<$ LOD & 3.7 & 0.22 & 0.05 \\
\hline & pellet 4 & 0.14 & 1.90 & 1.60 & 0.62 & 0.08 & 0.02 & $<$ LOD & 0.30 & 0.07 & $<$ LOD & 4.3 & 0.32 & 0.07 \\
\hline & strip & 0.07 & 0.77 & 0.57 & 0.20 & 0.01 & 0.07 & 5.3 & 0.26 & 9.2 & $<$ LOD & 1.6 & 5.6 & 9.2 \\
\hline & stick & $<$ LOD & 0.18 & 0.34 & 0.30 & 0.06 & 4.6 & 4.5 & 0.21 & 0.52 & $<$ LOD & 0.87 & 9.2 & 0.52 \\
\hline
\end{tabular}


Table 1. Continued.

\begin{tabular}{|c|c|c|c|c|c|c|c|c|c|c|c|c|c|c|}
\hline \multirow{2}{*}{$\begin{array}{l}\text { Category/ } \\
\text { Subcategory }\end{array}$} & \multirow{2}{*}{ Brand } & \multicolumn{5}{|c|}{ Sugars $^{a}$} & \multicolumn{3}{|c|}{ Alditols } & \multicolumn{2}{|c|}{ Humectants } & \multirow{2}{*}{$\begin{array}{l}\text { Total } \\
\text { Sugars }\end{array}$} & \multirow{2}{*}{$\begin{array}{c}\text { Total } \\
\text { Alditol }\end{array}$} & \multirow{2}{*}{$\begin{array}{c}\text { Total } \\
\text { Humectan }\end{array}$} \\
\hline & & Man & Fru & Glu & Suc & Mal & Xyl & Sor & Ino & Gly & PG & & & \\
\hline \multirow{4}{*}{$\begin{array}{l}\text { US tobacco } \\
\text { stick }\end{array}$} & 1 & $<$ LOD & 0.02 & 0.04 & 0.04 & 0.01 & 0.04 & 0.002 & 0.06 & $<$ LOD & $<$ LOD & 0.12 & 0.10 & $<$ LOD \\
\hline & 2 & $<$ LOD & 0.03 & 0.05 & 0.04 & 0.01 & 0.04 & 0.003 & 0.06 & $<$ LOD & $<$ LOD & 0.13 & 0.10 & $<$ LOD \\
\hline & 3 & $<$ LOD & 0.01 & 0.05 & 0.05 & 0.01 & 0.04 & 0.003 & 0.06 & $<$ LOD & 0.66 & 0.12 & 0.10 & 0.66 \\
\hline & 4 & $<$ LOD & 0.01 & 0.04 & 0.03 & 0.01 & 0.04 & 0.002 & 0.06 & $<$ LOD & $<$ LOD & 0.10 & 0.10 & $<$ LOD \\
\hline $\begin{array}{l}\text { IN creamy } \\
\text { snuff }\end{array}$ & & $<$ LOD & 0.03 & 0.09 & $<$ LOD & 0.03 & 0.08 & 22.5 & 0.03 & 0.24 & $<$ LOD & 0.14 & 22.6 & 0.24 \\
\hline $\begin{array}{l}\text { Ref. moist } \\
\text { snuff }(C R P 2)^{b}\end{array}$ & & $<$ LOD & $<$ LOD & $<$ LOD & $<$ LOD & $<$ LOD & 0.002 & $<$ LOD & 0.02 & $<$ LOD & $<$ LOD & $<$ LOD & 0.02 & $<$ LOD \\
\hline $\begin{array}{l}\text { Ref. dry snuff } \\
\text { (CRP3) }\end{array}$ & & $<$ LOD & $<$ LOD & $<$ LOD & $<$ LOD & $<$ LOD & 0.07 & $<$ LOD & 0.08 & $<$ LOD & $<$ LOD & $<$ LOD & 0.16 & $<$ LOD \\
\hline $\begin{array}{l}\text { Ref. loose } \\
\text { leaf (CRP4) }\end{array}$ & & 0.02 & 0.30 & 2.2 & 23.4 & 1.6 & 0.05 & 0.004 & 0.05 & 4.4 & $<$ LOD & 27.5 & 0.10 & 4.4 \\
\hline
\end{tabular}

a Man: mannose; Fru: fructose; Glu: glucose; Suc: sucrose; Mal: maltose; Xyl: xylitol; Sor: sorbitol; Ino; myo-inositol; Gly: glycerol; PG: propylene glycol

b CRP: CORESTA reference product

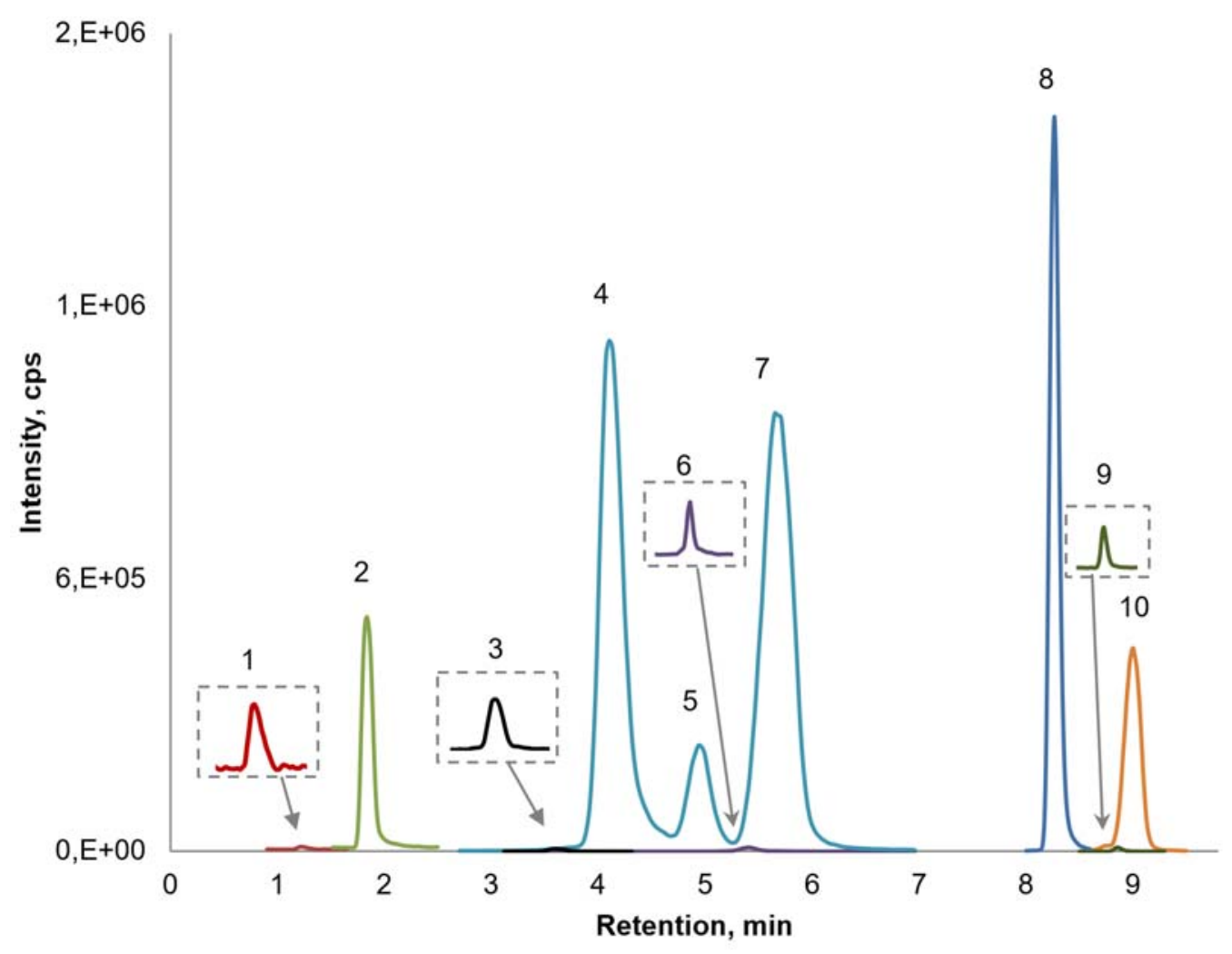

Figure 1. LC-MS/MS extracted ion chromatogram of sugars, alditols, and humectants in one US chewing plug sample.

1 - propylene glycol $(1.23 \mathrm{~min})$
2 - glycerol (1.81 $\mathrm{min})$
3 - xylitol (3.51 min)
4 - D-(-)-fructose $(4.06 \mathrm{~min})$
5 - D-(+)-mannose $(4.90 \mathrm{~min})$

Peaks $1,3,6$, and 9 are enlarged $200 \times$ and embedded in the graph.

$$
\begin{aligned}
& 6 \text { - D-sorbitol }(5.13 \mathrm{~min}) \\
& 7 \text { - D-(+)-glucose }(5.67 \mathrm{~min}) \\
& 8 \text { - sucrose }(8.26 \mathrm{~min}) \\
& 9 \text { - myo-inositol }(8.86 \mathrm{~min}) \\
& 10 \text { - maltose }(9.01 \mathrm{~min})
\end{aligned}
$$


a

Mean Sugar, $\%(w / w)$

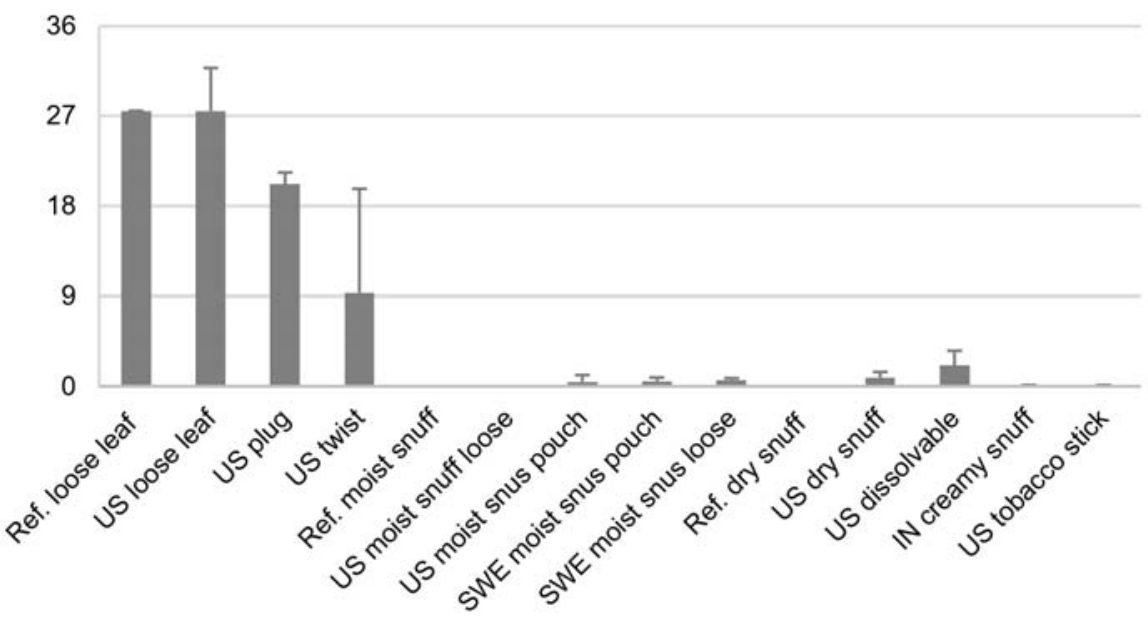

b

Mean Alditol, \%(w/w)

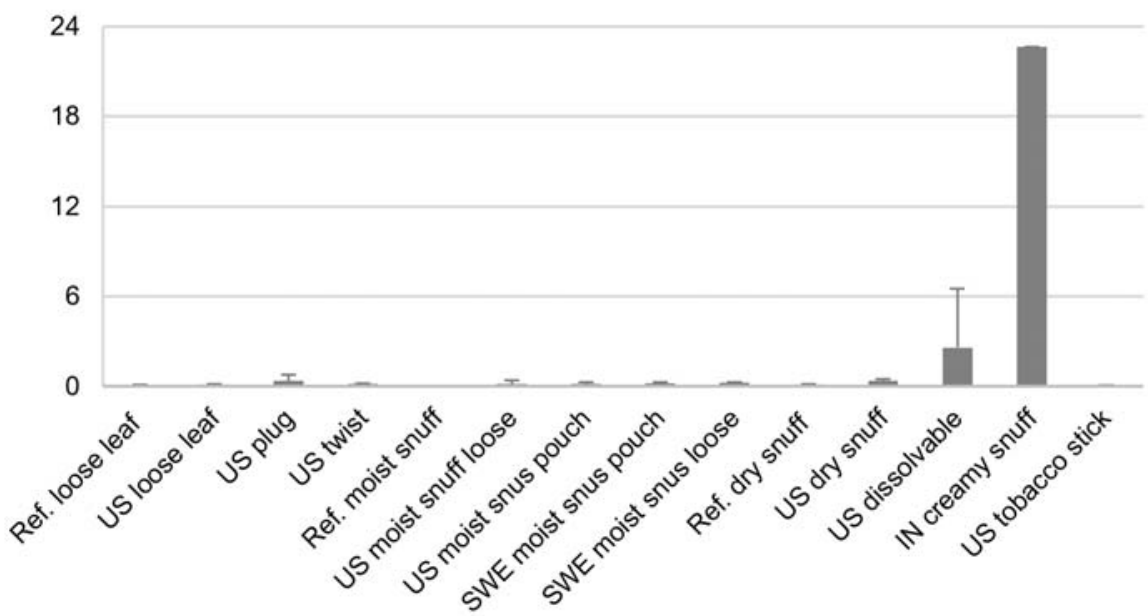

C

Mean Humectant, \%(w/w)

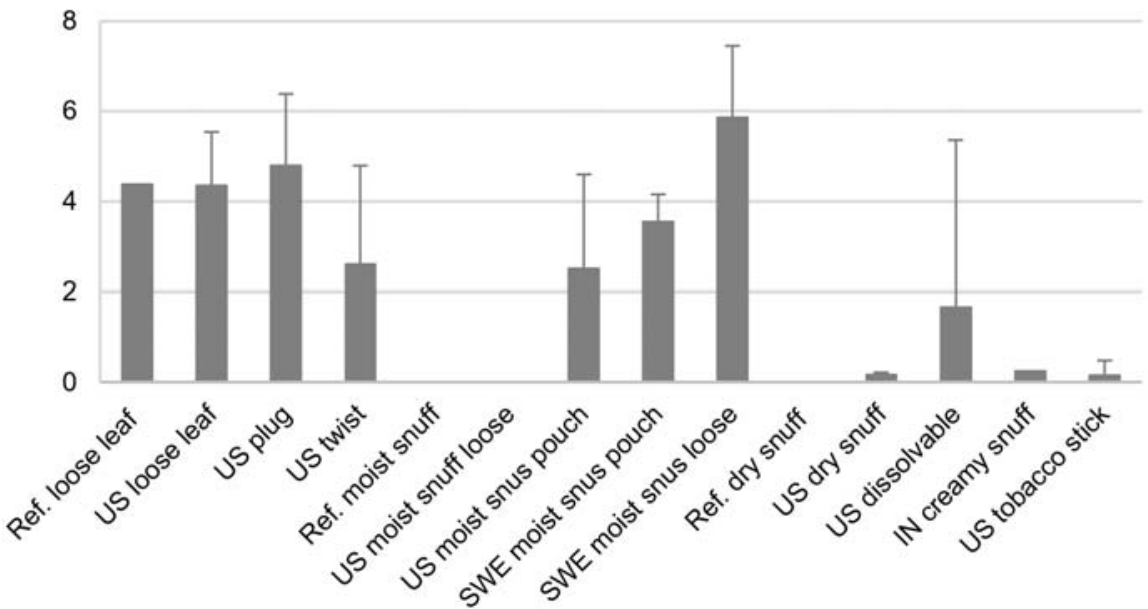

Figure 2. Total sugar (a), alditol (b), and humectant (c) for 11 categories/subcategories of ST. The error bars at the top of each column indicate the standard deviation of the measurement. SWE - Sweden; IN - India. 
Table 2. Mean values for sugars, alditols, and humectants measured in selected categories/subcategories of smokeless tobacco products. Unit: \% (mg/mg)

\begin{tabular}{|c|c|c|c|c|c|c|c|c|c|}
\hline \multirow{2}{*}{ Category/Subcategory } & \multicolumn{3}{|c|}{ Total Sugar } & \multicolumn{3}{|c|}{ Total Alditol } & \multicolumn{3}{|c|}{ Total Humectant } \\
\hline & \multicolumn{2}{|c|}{ Mean (range) } & \multirow{2}{*}{$\frac{S D^{a}}{4.3}$} & \multicolumn{2}{|c|}{ Mean (range) } & \multirow{2}{*}{$\begin{array}{l}\text { SD } \\
0.02\end{array}$} & \multicolumn{2}{|c|}{ Mean (range) } & \multirow{2}{*}{$\begin{array}{r}\text { SD } \\
1.2\end{array}$} \\
\hline US loose leaf $^{\text {b }}$ & 27.5 & $(21.0-33.4)$ & & 0.13 & $(0.09-0.14)$ & & 4.4 & $(3.2-6.4)$ & \\
\hline US plug & 20.2 & $(19.3-22.0)$ & 1.2 & 0.38 & $(0.10-1.1)$ & 0.40 & 4.8 & $(3.9-7.1)$ & 1.6 \\
\hline US twist & 9.3 & (0.06-20.6) & 10.4 & 0.16 & $(0.11-0.21)$ & 0.05 & 2.6 & $(0.08-4.0)$ & 2.2 \\
\hline US moist snuff (loose) & 0.01 & $(<\text { LOD }-0.05)^{\mathrm{C}}$ & 0.02 & 0.16 & $(<$ LOD-0.59) & 0.24 & $<$ LOD & & na ${ }^{d}$ \\
\hline US moist snus (pouch) & 0.44 & $(<$ LOD-1.7) & 0.70 & 0.17 & $(0.06-0.30)$ & 0.10 & 2.5 & $(<$ LOD-6.0) & 2.1 \\
\hline SWE moist snus (pouch) ${ }^{e}$ & 0.52 & $(0.25-1.2)$ & 0.36 & 0.22 & $(0.11-0.36)$ & 0.08 & 3.5 & $(2.4-4.0)$ & 0.61 \\
\hline SWE moist snus (loose) & 0.64 & $(0.33-0.78)$ & 0.21 & 0.24 & $(0.19-0.34)$ & 0.07 & 5.9 & $(3.6-7.0)$ & 1.6 \\
\hline US dry snuff & 0.86 & $(0.51-2.2)$ & 0.58 & 0.39 & $(0.32-0.66)$ & 0.12 & 0.17 & $(0.07-0.21)$ & 0.06 \\
\hline US dissolvable & 2.1 & $(0.87-4.3)$ & 1.5 & 2.6 & $(0.22-9.2)$ & 3.9 & 1.7 & $(0.05-9.2)$ & 3.7 \\
\hline Tobacco stick & 0.12 & $(0.10-0.13)$ & 0.01 & 0.10 & $(0.10-0.10)$ & 0.003 & 0.16 & (< LOD-0.66) & 0.33 \\
\hline IN creamy snuff ${ }^{f}$ & 0.14 & & na & 22.6 & & na & 0.24 & & na \\
\hline
\end{tabular}

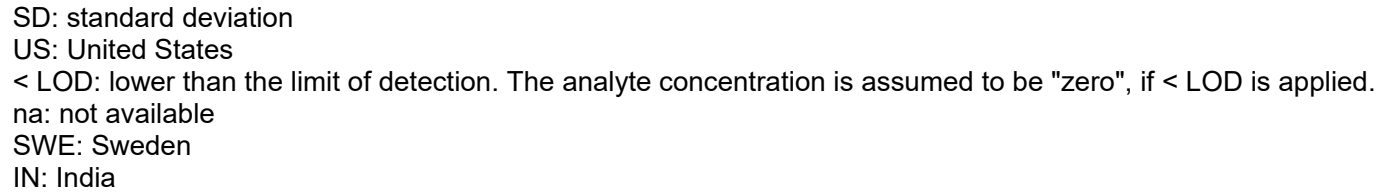

Mean total sugar, mean total alditol, and mean total humectant levels for chewing loose were all comparable to the corresponding values for reference chewing loose (CRP4, $4.4 \%$ ) (Tables 1 and 2). For US moist snuff loose, only one sample (\#6) had detectable glucose $(0.05 \%)$ and maltose $(0.004 \%)$; albeit at very low levels. The other moist snuff had no detectable sugar levels, which agrees with the values for the reference moist snuff (CRP2, < LOD) in Table 1. Regarding alditol levels for the same category, moist snuff (\#6) contained a relatively high alditol level $(0.59 \%)$, one sample contained no detectable alditol; whereas, the others had similar detected levels $(0.05-0.06 \%)$ slightly higher than the reference moist snuff (CRP2, 0.02\%) (Table 1). However, the results for dry snuff samples on mean total sugar, mean total alditol, and mean total humectant were not consistent with the reference dry snuff (CRP3) overall (Tables 1 and 2). All dry snuff samples had sugars $(0.51-2.2 \%)$ and humectant glycerol (0.07-0.21\%) detected, but reference dry snuff (CRP3, < LOD) had none detected; all dry snuff samples had relatively higher levels of alditol detected $(0.36-0.70 \%)$ compared to reference dry snuff (CRP3, $0.16 \%$ ) (Table 1).

Table 2 shows that chewing tobacco products had the highest mean total sugar contents ranging from 9.3-27.5\%. It is known that chewing tobacco, such as loose leaf and plug, has high levels of sugar because of the adding of sugar or different substances containing sugar (7). Among them, chewing loose had the highest mean total sugar content $(27.5 \%)$, followed by chewing plug with a mean total sugar content of $20.2 \%$, and chewing twist with the lowest mean total sugar content $9.3 \%$. Not all chewing twists were sweetened as shown in Table 1. Chewing twist (\#2) contained no detectable fructose, glucose, or mannose, and contained only very low sucrose and maltose contents $(0.02 \%$ and $0.04 \%$ respectively). Such low sugar content is comparable to the level of sugar existing in cigars and cured natural tobacco leaves $(38,39)$.

The formulations for SWE moist snus loose and pouch and for US moist snus pouch were different based on the measured total sugar, total alditol and total humectant levels. SWE moist snus loose had the highest measured values on all three parameters, followed by SWE moist snus pouch and US moist snus pouch. However, the sugar level of one US moist snus pouch sample (\#6) was much higher than most of the other snus samples. The humectant content of the same sample was also at the high level compared to the results of the other snus samples measured (Tables 1 and 2).

The sugar level in US moist snuff is non-detectable or very low, compared to US moist snus (Table 1), which could be explained by the tobacco pretreatment process. The tobacco leaf used for snuff usually experiences fermentation, but not for moist snus. The fermentation process used in products, such as moist snuff, may account for the loss of natural sugar (31). Considering the moisture levels for different samples (average levels: 53\% for moist snuff, $6 \%$ for dry snuff, $52 \%$ for Swedish snus, $26 \%$ for US moist snus), US dry snuff has a higher sugar level than US moist snuff and a similar level of sugar as SWE moist snus, based on the dry weight. It is possible that fermentation is less extensive for US dry snuff tobacco as compared to US moist snuff.

Three different subcategories (pellet, strip, stick) of dissolvable ST were examined in this study. Among them, pellet sample \#1 and \#2 are from one company, and \#3 and \#4 from another company. Pellets from different companies have the same levels of alditol and humectant but showed different levels of sugar. Only one strip and one stick sample were examined; the obtained results show they have relatively higher sugar, alditol and humectant levels than other forms of dissolvable ST (Table 1). Xylitol is suggested by dentist to be used as sugar substitute because it can decrease the dental cavity problem (40). The examined dissolvable stick sample had highest xylitol level $(4.6 \%)$ compared to all other samples $(0.32 \%)$ (Table 1$)$. The means and distribution ranges of mono- and disaccharides for each category or subcategory are summarized in 
Table 3. Mean values of measured monosaccharides and disaccharides in selected categories/subcategories of smokeless tobacco products. All values in $\%(\mathrm{mg} / \mathrm{mg})$.

\begin{tabular}{|c|c|c|c|c|c|c|c|c|}
\hline \multirow[t]{2}{*}{ Category/Subcategory } & \multicolumn{2}{|c|}{ Monosaccharides $^{a}$} & \multicolumn{2}{|c|}{ Disaccharides $^{a}$} & \multicolumn{2}{|c|}{$\begin{array}{l}\text { Monosaccharides } \\
\% \text { of total sugars }\end{array}$} & \multicolumn{2}{|c|}{$\begin{array}{c}\text { Disaccharides } \\
\% \text { of total sugars }\end{array}$} \\
\hline & Mean & (Range) & Mean & (Range) & Mean & (Range) & Mean & (Range) \\
\hline Ref. loose leaf & 2.5 & & 25 & & 9 & & 91 & \\
\hline US loose leaf & 11.6 & (3.9-24.3) & 15.9 & $(6.6-19.3)$ & 42 & $(19-79)$ & 58 & $(21-81)$ \\
\hline US plug & 6.9 & $(4.3-12.5)$ & 13.2 & $(7.2-16.1)$ & 34 & $(22-63)$ & 66 & $(37-78)$ \\
\hline US twist & 2.6 & $(<$ LOD-5.6) & 6.7 & $(0.06-15)$ & 28 & $(0-30)$ & 72 & $(70-100)$ \\
\hline US moist snuff (loose) & 0.01 & $(<$ LOD-0.05) & 0 & $(<$ LOD -0.001$)$ & $98^{\mathrm{C}}$ & & $2^{c}$ & \\
\hline US moist snus (pouch) & 0.16 & $(<$ LOD-0.65) & 0.20 & $(<$ LOD-1.0) & 44 & $(39-93)^{d}$ & 56 & $(7-61)^{d}$ \\
\hline SWE moist snus (pouch) & 0.44 & $(0.23-0.91)$ & 0.08 & $(0.02-0.31)$ & 85 & $(74-93)$ & 15 & $(7-26)$ \\
\hline SWE moist snus (loose) & 0.55 & $(0.09-0.76)$ & 0.09 & $(0.02-0.23)$ & 86 & $(29-97)$ & 14 & $(3-71)$ \\
\hline US dry snuff & 0.56 & $(0.09-1.42)$ & 0.30 & $(0.06-0.76)$ & 65 & $(18-91)$ & 35 & $(9-83)$ \\
\hline US dissolvable pellet & 1.9 & $(0.64-3.6)$ & 0.67 & $(0.48-0.99)$ & 68 & $(57-84)$ & 32 & $(16-43)$ \\
\hline US dissolvable strip & 1.4 & & 0.21 & & 87 & & 13 & \\
\hline US dissolvable stick & 0.52 & & 0.36 & & 59 & & 41 & \\
\hline Tobacco stick & 0.07 & $(0.06-0.08)$ & 0.05 & $(0.04-0.06)$ & 57 & $(52-62)$ & 43 & $(38-48)$ \\
\hline IN creamy snuff & 0.12 & & 0.03 & & 80 & & 20 & \\
\hline
\end{tabular}

a Monosaccharides: fructose, glucose, and mannose; Disaccharides: sucrose and maltose

b L LOD: lower than the limit of detection. The analyte concentration is assumed to be "zero", if < LOD is applied.

c only one US moist snuff (loose) had mono- and disaccharides detected at very low levels.

d one US moist snus (pouch) sample had no mono- and disaccharides detected.

Table 3. Figure 3 compares the mono- and disaccharide contents in each category or subcategory. SWE snus had relatively higher percentages of monosaccharides than disaccharides. The nature of the tobacco and the pretreatment process both could be reasons for this. If relatively large amounts of sugar or sugar containing substances are added to ST during the manufacturing process, the percentages of mono- and disaccharides in the detected total sugar in ST (e.g., US loose leaf, plug, and some twist products) are affected by their contents in the sugar additive sources. Figure 4 shows the significantly different addition of humectants glycerol and propylene glycol in different types of ST. The chewing products had much higher glycerol levels (2.4-4.0\% in mean) added than propylene glycol $(0.19-0.80 \%$ in mean). Snus products had similar levels of propylene glycol added $(2.5-3.5 \%$ in mean), however, the glycerol contents in different categories of snus were significantly different $(0.04-2.1 \%$ in mean). In US dry snuff, US pellet, US strip, US stick and India creamy snuff, only glycerol was detected. Only one

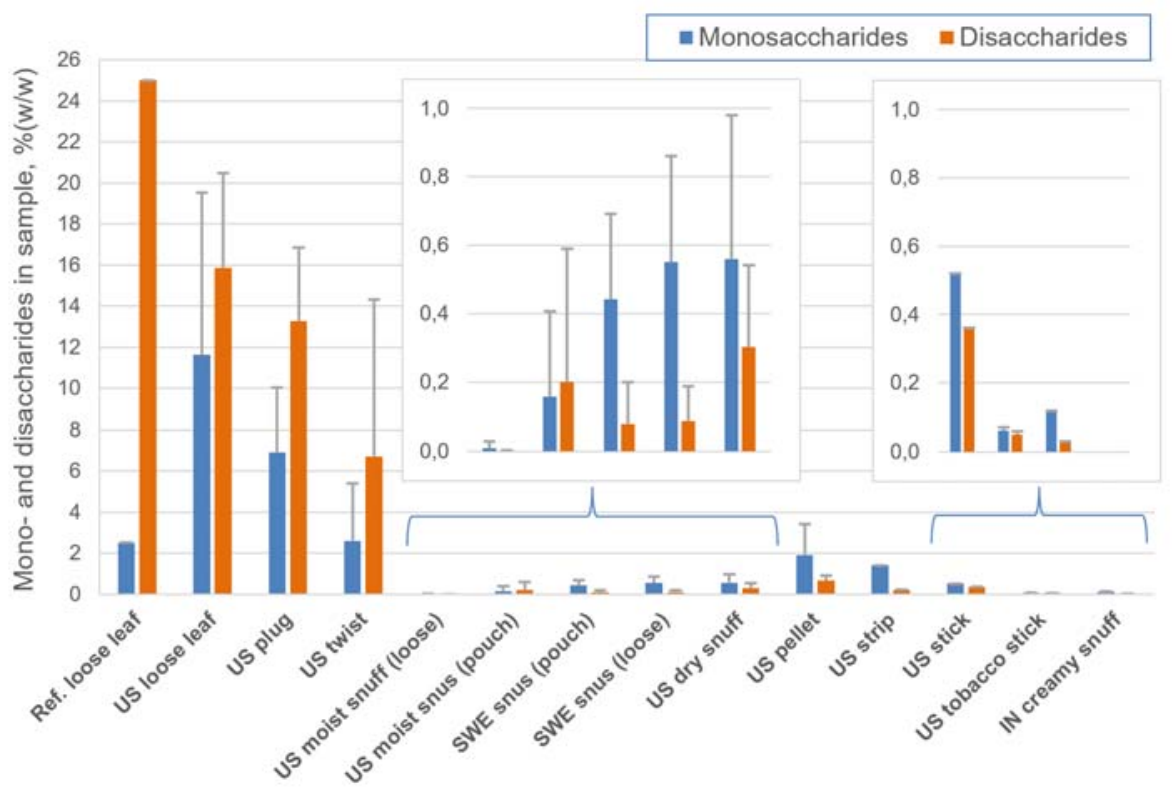

Figure 3. Comparison of mono- and disaccharide contents in each category or subcategory of ST. Reference dry and moist snuff had no sugar detected (not shown in the graph). 


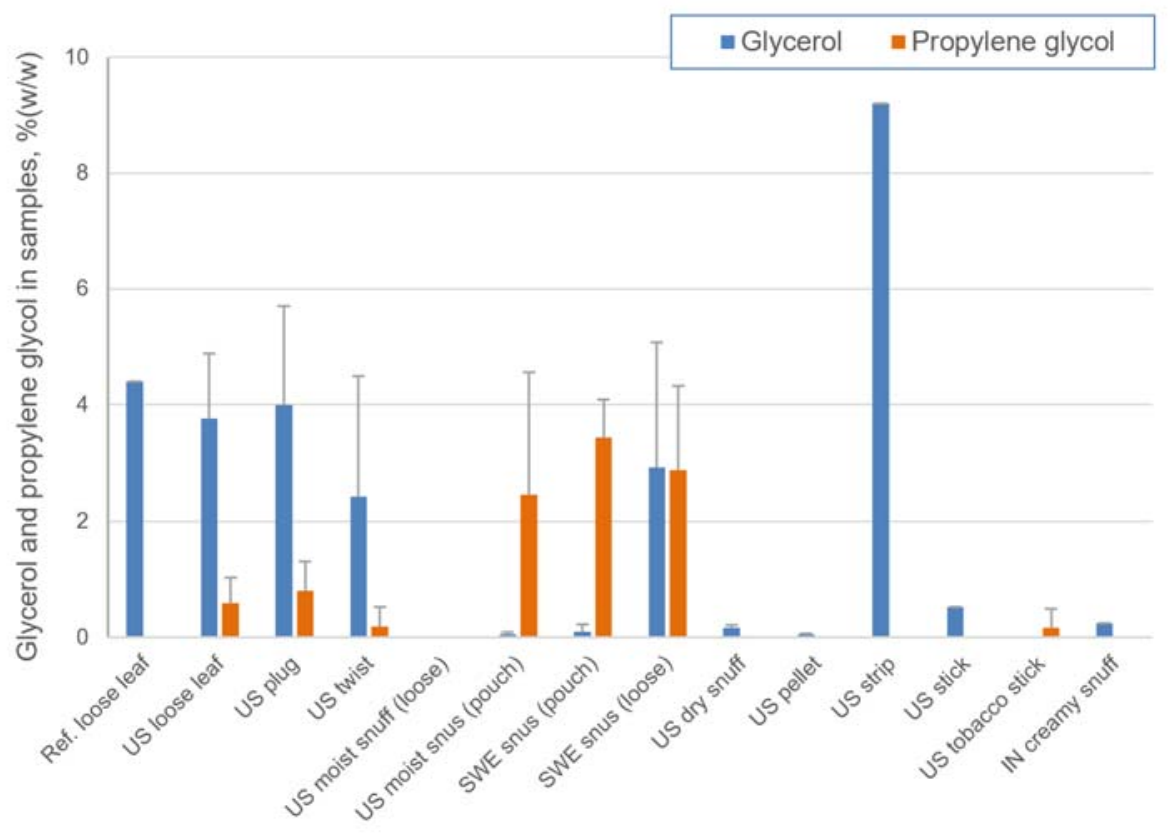

Figure 4. Comparison of glycerol and propylene glycol contents in each category or subcategory of ST. Reference dry and moist snuff had no humectants detected (not shown in the graph).

among four US tobacco stick samples had propylene glycol detected; all four same samples had no glycerol detected (Table 1). ST products have a broad variety of formulations (or categories/subcategories) of samples, and their mean total sugar and alcohol levels have a much broader span (0.05-27.5\% and $0.16-22.9 \%$ respectively) than smoking tobacco products $(0.6-5.8 \%$, and $1.9-3.3 \%$ respectively) including small cigars, cigarillos, and cigarettes, that we examined in our recent study (38). Though the mean values could possibly shift if more brands were analyzed, the results obtained with a limited number of examined samples in this study give an informative view of product constituents. The work presented in this manuscript is complementary to our previous work on smokeless tobacco products $(7,36,37)$ Characterization of these products is helpful for a better understanding of these products and aids other researchers and public health advocates investigating the risk of using smokeless tobacco products.

\section{ACKNOWLEDGEMENT}

The authors gracefully thank Ms. Tameka Lawler for providing partial smokeless products.

\section{REFERENCES}

1. Boffetta, P., S. Hecht, N. Gray, P. Gupta, and K. Straif: Smokeless Tobacco and Cancer; Lancet Oncol. 9 (2008) 667-675.

DOI: 10.1016/S1470-2045(08)70173-6

2. Grøtvedt, L., L. Forsén, K. Stavern, and S. GraffIversen: Patterns of Snus and Cigarette Use: A Study of Norwegian Men Followed From Age 16 to 19; Tob.
Control 22 (2013) 382-388.

DOI: $10.1136 /$ tobaccocontrol-2011-050158

3. Bhattacharyya, N.: Trends in the Use of Smokeless Tobacco in United States, 2000-2010; Laryngoscope 122 (2012) 2175-2178. DOI: 10.1002/lary.23448

4. Delnevo, C.D., O.A. Wackowski, D.P. Giovenco, M. T. Bover Manderski, M. Hrywna, and P.M. Ling: Examining Market Trends in the United States Smokeless Tobacco Use: 2005-2011; Tob. Control 23 (2014) 107-112. DOI: 10.1136/tobaccocontrol-2012-050739

5. Willis, D., M. Popovech, F. Gany, and J. Zelikoff: Toxicology of Smokeless Tobacco: Implications for Immune, Reproductive, and Cardiovascular Systems; J. Toxicol. Environ. Health B Crit Rev (2012) 317-331. DOI: $10.1080 / 10937404.2012 .689553$

6. International Agency for Research on Cancer (IARC): Smokeless Tobacco and Some Tobacco-Specific $\mathrm{N}$-Nitrosamines; IARC Monographs on the Evaluation of Carcinogenic Risks to Humans, Vol 89, IARC, Lyon, France, 2008.

7. Lawler, T.S., S.B. Stanfill , L. Zhang, D.L. Ashley, and C.H. Watson: Chemical Characterization of Domestic Oral Tobacco Products: Total Nicotine, $\mathrm{pH}$, Unprotonated Nicotine and Tobacco-Specific $N$-Nitrosamines; Food Chem. Toxicol. 57 (2013) 380-386. DOI: $10.1016 /$ j.fct.2013.03.011

8. Boffetta, P. and K. Straif: Use of Smokeless Tobacco and Risk of Myocardial Infarction and Stroke: Systematic Review with Meta-Analysis; BMJ 339 (2009) b3060. DOI: 10.1136/bmj.b3060

9. Bolinder, G., L. Alfredsson, A. Englund, and U. de Faire: Smokeless Tobacco Use and Increased Cardiovascular Mortality Among Swedish Construction Workers; Am. J. Public Health 84 (1994) 399-404.

10. Gupta, R., H. Gurm, and J.R. Bartholomew: Smokeless 
Tobacco and Cardiovascular Risk; Arch. Intern. Med. 164 (2004) 1845-1849.

DOI: 10.1001/archinte.164.17.1845

11. Foulds, J., L. Ramström, M. Burke, and K. Fagerström: Effect of Smokeless Tobacco (Snus) on Smoking and Public Health in Sweden; Tob. Control 12 (2002) 349-359. DOI: 10.1136/tc.12.4.349

12. Östenson, C.G., A. Hilding, V. Grill, and S. Efendic: High Consumption of Smokeless Tobacco ("Snus") Predicts Increased Risk of Type 2 Diabetes in a 10-Year Prospective Study of Middle-Aged Swedish Men; Scand. J. Public Health 40 (2012) 730-737.

DOI: $10.1177 / 1403494812459814$

13. Fisher, M.A., G.W. Taylor, and K.R. Tilashalski: Smokeless Tobacco and Severe Active Periodontal Disease, NHANES III; J. Dent. Res. 84 (2005) 705-710. DOI: 10.1177/154405910508400804

14. Holmen, A., U. Stromberg, K. Magnusson, and S. Twetman: Tobacco Use and Caries Risk Among Adolescents - A Longitudinal Study in Sweden; BMC Oral Health 13 (2013) 31.

DOI: $10.1186 / 1472-6831-13-31$

15. Tomar, S.L. and D.M. Winn: Chewing Tobacco Use and Dental Caries Among U.S. Men; J. Am. Dent. Assoc. 130 (1999) 1601-1610.

DOI: 10.14219/jada.archive.1999.0099

16. Luo, J., W. Ye, K. Zendehdel, J. Adami, H.O. Adami, P. Boffetta, and O. Nyrén: Oral Use of Swedish Moist Snuff (Snus) and Risk for Cancer of the Mouth, Lung, and Pancreas in Male Construction Workers: A Retrospective Cohort Study; Lancet 369 (2007) 2015-2020. DOI: 10.1016/S0140-6736(07)60678-3

17. Weitkunat, R., E. Sanders, and P.N. Lee: Meta-Analysis of the Relation Between European and American Smokeless Tobacco and Oral Cancer; BMC Public Health 7 (2007) 334. DOI: 10.1186/1471-2458-7-334

18. Zhou, J., D.S. Michaud, S.M. Langevin, M.D. McClean, M. Eliot, and K.T. Kelsey: Smokeless Tobacco and Risk of Head and Neck Cancer: Evidence From a Case-Control Study in New England; Int. J. Cancer 132 (2013) 1911-1917. DOI: 10.1002/ijc.27839

19. Cotti, E., C. Dessi, A. Piras, and G. Mercuro: Can a Chronic Dental Infection Be Considered a Cause of Cardiovascular Disease? A Review of the Literature; Int. J. Cardiol. 148 (2011) 4-10.

DOI: $10.1016 /$ j.ijcard.2010.08.011

20. Desvarieux, M., R.T. Demmer, D.R. Jacobs Jr, T. Rundek, B. Boden-Albala, R.L. Sacco, and P.N. Papapanou: Periodontal Bacteria and Hypertension: The Oral Infections and Vascular Disease Epidemiology Study (INVEST); J. Hypertens. 28 (2010) 1413-1421. DOI: $10.1097 /$ HJH.0b013e328338cd36

21. Genco, R., S. Offenbacher, and J. Beck: Periodontal Disease and Cardiovascular Disease: Epidemiology and Possible Mechanisms; J. Am Dent. Assoc. 133 Suppl. (2002) 14S-22S.

DOI: 10.14219/jada.archive.2002.0375

22. Lockhart, P.B., A.F. Bolger, P.N. Papapanou, O. Osinbowale, M. Trevisan, M.E. Levison, K.A. Taubert, J.W. Newburger, H.L. Gornik, M.H. Gewitz, W.R. Wilson, S.C. Smith Jr, and L. M. Baddour on behalf of the American Heart Association Rheumatic Fever,
Endocarditis, and Kawasaki Disease Committee of the Council on Cardiovascular Disease in the Young, Council on Epidemiology and Prevention, Council on Peripheral Vascular Disease, and Council on Clinical Cardiology: Periodontal Disease and Atherosclerotic Vascular Disease: Does the Evidence Support an Independent Association? A Scientific Statement From the American Heart Association; Circulation 125 (2012) 2520-2544. DOI: 10.1161/CIR.0b013e31825719f3

23. Roosaar, A., A.L. Johansson, G. Sandborgh-Englund, T. Axéll, and O. Nyrén: Cancer and Mortality Among Users and Nonusers of Snus; Int. J. Cancer 123 (2008) 168-173. DOI: 10.1002/ijc.23469.

24. Melikian, A.A. and D. Hoffmann: Smokeless Tobacco: A Gateway to Smoking or a Way Away From Smoking; Biomarkers 14 (2009) 85-89. DOI: $10.1080 / 13547500902965401$.

25. Critchley, J.A. and B. Unal: Health Effects Associated with Smokeless Tobacco: A Systematic Review; Thorax 58 (2003) 435-443.

DOI: $10.1136 /$ thorax.58.5.435

26. Hatsukami, D.K., C. Lemmonds, and S.L. Tomar: Smokeless Tobacco Use: Harm Reduction or Induction Approach?; Prev. Med. 38 (2004) 309-317. DOI: 10.1016/j.ypmed.2003.10.006

27. Severson, H.H., K.K. Forrester, and A. Biglan: Use of Smokeless Tobacco is a Risk Factor for Cigarette Smoking; Nicotine Tob. Res. 9 (2007) 1331-1337. DOI: $10.1080 / 14622200701705209$

28. Tomar, S.L.: Is Use of Smokeless Tobacco a Risk Factor for Cigarette Smoking? The U.S. Experience; Nicotine Tob. Res. 5 (2003) 561-569.

29. National Cancer Institute (NCI): Monograph 2: Smokeless Tobacco or Health: An International Perspective. Available at: http://cancercontrol.cancer.gov/brp/tcrb/ monographs/2/index.html (accessed Feb 13, 2019).

30. Talhout, R., A. Opperhuizen, and J.G.C. van Amsterdam: Sugars as Tobacco Ingredient: Effects on Mainstream Smoke Composition; Food Chem. Toxicol. 44 (2006) 1789-1798. DOI: 10.1016/j.fct.2006.06.016

31. Di Giacomo, M., M. Paolino, D. Silvestro, G. Vigliotta, F. Imperi, P. Visca, P. Alifano, and D. Parente: Microbial Community Structure and Dynamics of Dark FireCured Tobacco Fermentation; Appl. Environ. Microbiol. 73 (2007) 825-837.

DOI: 10.1128/AEM.02378-06

32. Andersen, R.A., P.D. Fleming, H.R. Burton, T.R. Hamilton-Kemp, and T.G. Sutton: Nitrosated, Acylated, and Oxidized Pyridine Alkaloids during Storage of Smokeless Tobaccos - Effects of Moisture, Temperature, and Their Interactions; J. Agric. Food Chem. 39 (1991) 1280-1287. DOI: 10.1021/jf00007a017

33. Hsu, S.C., R.L. Pollack, A.-F.C. Hsu, and R.E. Going: Sugars Present in Tobacco Extracts; J. Am. Dent. Assoc. 101 (1980) 915-918. DOI: 10.14219/jada.archive.1980.0465

34. Clarke, M.B., D.Z. Bezabeh, and C.T. Howard: Determination of Carbohydrates in Tobacco Products by Liquid Chromatography-Mass Spectrometry/Mass Spectrometry: A Comparison with Ion Chromatography and Application to Product Discrimination; J. Agric. Food Chem. 54 (2006) 1975-1981. 
DOI: $10.1021 /$ jf052925+

35. Rainey, C.L., J.R. Shifflett, J.V. Goodpaster, and D.Z. Bezabeh: Quantitative Analysis of Humectants in Tobacco Products Using Gas Chromatography (GC) with Simultaneous Mass Spectrometry (MSD) and Flame Ionization Detection (FID); Beitr. Tabakforsch. Int. 25 (2013) 576-585. DOI: 10.2478/cttr-2013-0934

36. Richter, P., K. Hodge, S. Stanfill, L. Zhang, and C. Watson: Surveillance of Moist Snuff: Total Nicotine, Moisture, pH, Un-Ionized Nicotine, and TobaccoSpecific Nitrosamines; Nicotine Tob. Res. 10 (2008) 1645-1652. DOI: 10.1080/14622200802412937

37. Stanfill, S.B., G.N. Connolly, L. Zhang, L.T. Jia, J.E. Henningfield, P. Richter, T.S. Lawler, O.A. Ayo-Yusuf, D.L. Ashley, and C.H.Watson: Global Surveillance of Oral Tobacco Products: Total Nicotine, Unionised Nicotine and Tobacco-Specific $N$-Nitrosamines; Tob. Control 20 (2011) e2. DOI: 10.1136/tc.2010.037465

38. Wang, L., R. Bravo Cardenas, and C.H. Watson: An Isotope Dilution Ultra High Perfomance Liquid Chromatography-Tandem Mass Spectrometry Method for the Simultaneous Determination of Sugars and Humectants in Tobacco Products; J. Chromatogr. A 1514C (2017) 95-102. DOI: 10.1016/j.chroma.2017.07.079

39. Shifflett, J.R., L.A. Jones, E.R. Limowski, and D.Z. Bezabeh: Comparison of Segmented Flow Analysis and Ion Chromatography for the Quantitative Characterization of Carbohydrates in Tobacco Products; J. Agric. Food Chem. 60 (2012) 11714-11722.

DOI: $10.1021 /$ jf303421n
40. Alanen, P., P. Isokangas, and K. Gutmann: Xylitol Candies in Caries Prevention: Results of a Field Study in Estonian Children; Community Dent. Oral. Epidemiol. 28 (2000) 218-224.

DOI: $10.1034 /$ j.1600-0528.2000.280308.x

Corresponding author:

Roberto Bravo Cardenas, Ph.D. Department of Health and Human Services U.S. Centers for Disease Control and Prevention National Center for Environmental Health Division of Laboratory Sciences Tobacco and Volatiles Branch 4770 Buford Highway NE Atlanta 30341, GA, USA

Telephone: +1 770-488-7896

E-mail: rbravo@cdc.gov 